\title{
The use of splitting methods in the numerical simulation of reacting flows *
}

\author{
A. Araújo, J.A. Ferreira, P. Oliveira, F. Patrício \\ Departamento de Matemática \\ Universidade de Coimbra \\ Portugal \\ P. Rosa \\ Departamento de Física Matemática \\ Instituto Superior de Engenharia de Coimbra \\ Portugal
}

December 20, 2002

\begin{abstract}
The aim of this paper is to study discretizations of convectiondiffusion-reaction equations using splitting methods. Estimates for the physical splitting errors and the numerical splitting errors are established. These estimates lead to the selection of optimal sequences and coupling of physical phenomena and adequate use of implicitness and explicitness. Numerical simulations of two chemical industry problems are included.
\end{abstract}

\section{Introduction}

Numerical simulation of reacting flows is required in all problems coming from the chemical industry, and also in the modelling of certain regions of hypersonic aerodynamics. The added complexity of this kind of problems comes from the fact that often a wide range of time scales is present. This leads to numerical difficulties related to the possible stiffness of reaction

*This work has been supported by Centro de Matemática da Universidade de Coimbra and Project POCTI/35039/MAT/2000 
terms. Considering the Partial Differential Equations that describe reacting flows, we may split them additively into advective transport, diffusive transport and chemical transformations. We thus obtain submodels corresponding to different physical phenomena,that are easier to solve separately. Recent literature $([2],[4],[6])$ has provided several ways of dealing with operator splitting as far as the sequence of these physical phenomena and also the possible coupling between some of them are concerned. Numerical simulations in $[0,1]$ pointed out that the coupling of reactions with diffusive transport in a sequence composed by a convection problem in a certain interval $[0$, $\mathrm{t} / 2]$, a diffusion-reaction problem in $[0, \mathrm{t}]$ and a final convection problem in $[t / 2, t]$ perform notably better than an uncoupled splitting. In this paper we present a theoretical justification of this numerical evidence by studying the splitting errors and the stability properties of some numerical methods, based on different sequences and couplings of physical phenomena.

The splitting error has two main contributions: a physical splitting error that would exist even if submodels were solved exactly, and a numerical splitting error, related with the approximation of each submodel and the way these submodels are linked. In section 2 we establish the order of the physical splitting error for different sequences and couplings of the phenomena. In section 3 we show how the stability properties of numerical splitting errors based on the previous sequences and couplings, depend on the alternating use of implicit and explicit methods. Numerical simulations of two chemical industry problems will be presented in section 4 . Namely we exhibit results concerning a fixed bed catalytic reactor and a paper industry digester.

\section{Splitting methods and splitting errors}

Let us consider convection-diffusion-reaction equations of type

$$
\frac{\partial c}{\partial t}(z, t)=F_{1}(c, z, t)+F_{2}(c, z, t)+F_{3}(c, z, t)
$$

where $c$ denotes a specie concentration and $F_{1}(c, z, t), F_{2}(c, z, t), F_{3}(c, z, t)$ represent respectively the convection, the diffusion and the reaction terms.

Let us define in $[0, T]$ the splitting grid $\left\{t_{s}\right\}$ with $t_{s}=s \Delta t$ and $t_{s+1 / 2}=$ $t_{s}+\Delta t / 2$ where $\Delta t$ denotes the splitting step size. We suppose that the concentration $c$ at $t=t_{s}$ is known ( at least approximately ). The computation of an approximation of $c$ at time level $t_{s+1}$ can be obtained using several splitting algorithms. In this paper we analyse from a theoretical viewpoint two functional splitting procedures, suggested in [6], to study an atmospheric pollution problem. 


\section{- Functional splitting I}

Problem (1) is decomposed in five subproblems respectively (Convection, Diffusion, Reaction, Diffusion, Convection) as follows:

$$
\begin{gathered}
\frac{\partial u}{\partial t}(z, t)=F_{1}(u, z, t), t \in\left[t_{s}, t_{s+1 / 2}\right], \quad u\left(t_{s}\right)=c\left(t_{s}\right), \\
\frac{\partial v}{\partial t}(z, t)=F_{2}(v, z, t), t \in\left[t_{s}, t_{s+1 / 2}\right], \quad v\left(t_{s}\right)=u\left(t_{s+1 / 2}\right), \\
\frac{\partial w}{\partial t}(z, t)=F_{3}(w, z, t), t \in\left[t_{s}, t_{s+1}\right], \quad w\left(t_{s}\right)=v\left(t_{s+1 / 2}\right), \\
\frac{\partial v^{*}}{\partial t}(z, t)=F_{2}\left(v^{*}, z, t\right), t \in\left[t_{s+1 / 2}, t_{s+1}\right], \quad v^{*}\left(t_{s+1 / 2}\right)=w\left(t_{s+1}\right), \\
\frac{\partial u^{*}}{\partial t}(z, t)=F_{1}\left(u^{*}, z, t\right), t \in\left[t_{s+1 / 2}, t_{s+1}\right], \quad u^{*}\left(t_{s+1 / 2}\right)=v^{*}\left(t_{s+1}\right) .
\end{gathered}
$$

The splitting solution I at $t_{s+1}$ is $u^{*}\left(t_{s+1}\right)$ - the exact solution of (2)-(6) - which represents an approximation of $c\left(t_{s+1}\right)$.

\section{- Functional splitting II}

Problem (1) is decomposed in three subproblems (Convection, Diffusion+Reaction, Convection) as described below

$$
\begin{gathered}
\frac{\partial u}{\partial t}(z, t)=F_{1}(u, z, t), t \in\left[t_{s}, t_{s+1 / 2}\right], \quad u\left(t_{s}\right)=c\left(t_{s}\right), \\
\frac{\partial v}{\partial t}(z, t)=F_{2}(v, z, t)+F_{3}(v, z, t), t \in\left[t_{s}, t_{s+1}\right], \quad v\left(t_{s}\right)=u\left(t_{s+1 / 2}\right), \\
\frac{\partial u^{*}}{\partial t}(z, t)=F_{1}\left(u^{*}, z, t\right), t \in\left[t_{s+1 / 2}, t_{s+1}\right], \quad u^{*}\left(t_{s+1 / 2}\right)=v^{*}\left(t_{s+1}\right) .
\end{gathered}
$$

The splitting solution II at $t_{s+1}$ is $u^{*}\left(z, t_{s+1}\right)$ - the exact solution of (7)-(9) - which stands for an approximation of $c\left(t_{s+1}\right)$. 
Let us represent by $c_{I}$ and $c_{I I}$ respectively the splitting solutions I and II. In the case operators $F_{1}, F_{2}$ are linear and $F_{3}$ depends only on $z$ we can estimate the splitting errors $\left\|c-c_{I}\right\|_{\infty}$ and $\left\|c-c_{I I}\right\|_{\infty}$.

Proposition 1 Let $F_{1}(c, z, t)=-\alpha \frac{\partial c}{\partial z}(z, t), \quad F_{2}(c, z, t)=\beta \frac{\partial^{2} c}{\partial z^{2}}(z, t), \quad$ and $F_{3}(c, z, t)=F_{3}(z)$, with $\left|F_{3}^{\prime}\right| \leq q, c(z, 0)=c_{0}(z)$ and

$$
\lim _{z \rightarrow \pm \infty} c(z, t)=0
$$

for all $t$. Then

$$
\left\|c-c_{I}\right\|_{\infty}=O\left(\Delta t^{3 / 2}\right)
$$

and

$$
\left\|c-c_{I I}\right\|_{\infty}=O\left(\Delta t^{2}\right)
$$

Proof: Using Fourier transforms we have for the exact solution of (1)

$c(z, t)=\frac{1}{\sqrt{\pi}} \int_{0}^{t} \int_{\mathbb{R}} F_{3}(z-\alpha \xi+2 \sqrt{\beta \xi} y) e^{-y^{2}} d y d \xi+\frac{1}{\sqrt{\pi}} \int_{\mathbb{R}} c_{0}(z-\alpha t+2 \sqrt{\beta t} \xi) e^{-\xi^{2}} d \xi$.

It is a tedious but straightforward task to establish that the splitting solutions $c_{I}$ and $c_{I I}$ are given respectively by

$$
\begin{aligned}
& c_{I}(z, t)=\frac{1}{\sqrt{\pi}} \int_{\mathbb{R}} t F_{3}\left(z-\frac{\alpha t}{2}+2 \sqrt{\frac{\beta t}{2}} y\right) e^{-y^{2}} d y+\frac{1}{\sqrt{\pi}} \int_{\mathbb{R}} c_{0}(z-\alpha t+2 \sqrt{\beta t} \xi) e^{-\xi^{2}} d \xi, \\
& c_{I I}(z, t)=\frac{1}{\sqrt{\pi}} \int_{0}^{t} \int_{\mathbb{R}} F_{3}\left(z-\frac{\alpha t}{2}+2 \sqrt{\beta \xi} y\right) e^{-y^{2}} d y d \xi+\frac{1}{\sqrt{\pi}} \int_{\mathbb{R}} c_{0}(z-\alpha t+2 \sqrt{\beta t} \xi) e^{-\xi^{2}} d \xi .
\end{aligned}
$$

From (10) and (11) we have

$$
\left|c(z, t)-c_{I}(z, t)\right| \leq \frac{q}{\sqrt{\pi}}\left|\int_{\mathbb{R}} \int_{0}^{t}\left(\alpha \xi-\frac{\alpha k}{2}+2 \sqrt{\beta \xi}-\sqrt{\frac{\beta t}{2}} y\right) e^{-y^{2}} d y d \xi\right|
$$

and considering that $\int_{\mathbb{R}} e^{-y^{2}} d y=\sqrt{\pi}$ and $\int_{\mathbb{R}} e^{-y^{2}}|y| d y=1$ we easily conclude

$$
\left\|c-c_{I}\right\|_{\infty}=O(\Delta t \sqrt{\Delta t}) .
$$

From (10) and (12) we conclude

$$
\left|c(z, t)-c_{I I}(z, t)\right| \leq \frac{q}{\sqrt{\pi}}\left|\int_{\mathbb{R}} \int_{0}^{t}\left(\alpha \xi-\frac{\alpha t}{2} y\right) e^{-y^{2}} d y d \xi\right|
$$


and consequently

$$
\left\|c-c_{I I}\right\|_{\infty}=O\left(\Delta t^{2}\right)
$$

We note that the integral terms in (11) and (12), concerning the initial conditions $c_{0}(z)$, introduce no error in $c_{I}$ and $c_{I I}$. Also if $F_{3}$ is a linear function of $z$ then $c_{I}=c_{I I}$.

In [6], the authors, while studying an atmospheric problem, point out that splitting II lead to more accurate numerical results. The estimates of Proposition 1 can justify from a theoretical point of view this numerical evidence.

The following example illustrates the estimates of Proposition 1. Let us consider the initial boundary value problem

$$
\frac{\partial c}{\partial t}(z, t)=\gamma_{1} \frac{\partial c}{\partial z}(z, t)+\gamma_{2} \frac{\partial^{2} c}{\partial z^{2}}(z, t)+\gamma_{3}\left(c(z, t)-c_{w}(t)\right)+\gamma_{4} r(c)
$$

with initial boundary conditions

$$
\left\{\begin{array}{l}
c(z, 0)=f(z), z \in(0,1) \\
c(0,0)=c_{F}(0) \\
\gamma_{2} \frac{\partial c}{\partial z}(0, t)=\left[c(0, t)-c_{F}(t)\right] \gamma_{1}, t>0 \\
\frac{\partial c}{\partial z}(1, t)=0, t \geq 0
\end{array}\right.
$$

In (13), $\gamma_{i}, i=1, \ldots, 5$, are positive constants, $r(c)$ and $c_{w}(t)$ represent known functions. In (14), $f$ and $c_{F}$ are also assumed to be known.

We took in (14), $r(c)=c^{2}, \gamma_{1}=-1, \gamma_{2}=10^{-3}, \gamma_{3}=-0.5, \gamma_{4}=0.5$, $f(z)=1.11067+0.359664 z+0.325977 z^{2}$ and $c_{w}(t)=0.8$.

In Figure 1 are plotted the exact and numerical splitting solutions for $t=0.5$ and $t=1$. We note that splitting II solution is much more accurate than splitting solution I.

In Proposition 1 we consider a convection-diffusion-reaction problem in an unbounded domain. In the case of convection-reaction equations estimates have been established under a boundary condition along $z=0$. 

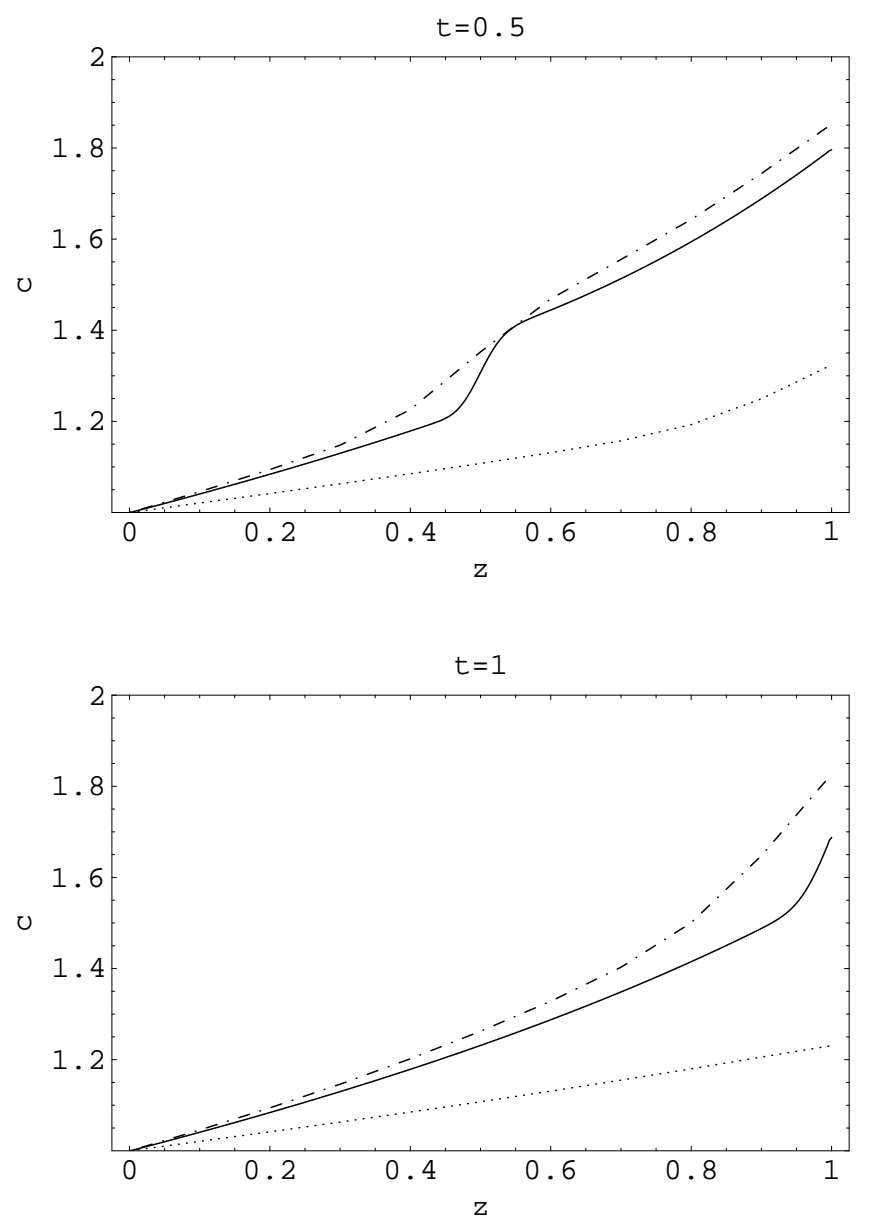

Figure 1: Numerical solutions (split I: dots; split II: dash-dots) and exact solution for (13)-(14). 
Proposition 2 Let $F_{2}=0$ and $F_{3}(c, z, t)=F_{3}(c)$, with $\left|F_{3}^{\prime}\right| \leq q$.

1. If $c(z, 0)=c_{0}(z)$ for $z \in(0,+\infty)$ and $c(0, t)=g(t)$ with $c_{0}$ and $g$ enough smooth functions, then:

(a) if $F_{1}(c)=\alpha(z, t) \frac{\partial c}{\partial z}$ we have $c_{I I}=c$ for $z>\alpha$ t and $\left\|c-c_{I I}\right\|_{\infty}=$ $O(\Delta t)$ for $z \leq \alpha t$

(b) if $F_{1}(c)=\frac{\partial}{\partial z} f(c)$ we have $\left\|c-c_{I I}\right\|_{\infty}=O(\Delta t)$.

2. If $c(z, 0)=c_{0}(z), z \in \mathbb{R}$, then the splitting solution is the exact solution.

Proof: We just present the proof of $1(a)$. The exact solution satisfies

$$
\left\{\begin{array}{l}
\int_{g\left(\frac{\alpha t-z}{\alpha}\right)}^{c(z, t)} \frac{d c}{F_{3}(c)}=\frac{z}{\alpha}, \quad z \leq \alpha t, \\
\int_{c_{0}(z-\alpha t)}^{c(z, t)} \frac{d c}{F_{3}(c)}=t, \quad z>\alpha t .
\end{array}\right.
$$

The solution $c_{I I}$ of (7)- (9) verifies

$$
\begin{cases}c(z, t)=g\left(\frac{\alpha t-z}{\alpha}\right), & 0 \leq z \leq \frac{\alpha t}{2} \\ \int_{g\left(\frac{\alpha t-z}{\alpha}\right)}^{c(z, t)} \frac{d c}{F_{3}(c)}=\frac{z}{\alpha}, & \frac{\alpha t}{2}<z \leq \alpha t, \\ \int_{c_{0}(z-\alpha t)}^{c(z, t)} \frac{d c}{F_{3}(c)}=t, \quad z>\alpha t .\end{cases}
$$

From (15) and (16) the result follows.

We observe that if no boundary conditions are considered in the convection-reaction problem, as in 2. of Proposition 2, the exact solution is obtained. When a boundary condition is considered as in 1 . of Proposition 1 an error of order $\Delta t$ propagates in the domain defined by $z \leq \alpha t$.

\section{$3 \quad$ Numerical splitting methods}

\subsection{General description of the family of methods}

We consider, in what follows, numerical methods obtained by the discretization of (7)-(9). In the interval $[0, T]$ we consider the splitting grid $\left\{t_{s}\right\}$ and in the space domain we define the grid $\left\{z_{j}\right\}$ with $z_{j}-z_{j-1}=h$. Let $U_{h}^{s}, V_{h}^{s}$ and 
$U_{h}^{s *}$ be numerical approximations at $t_{s}$ of $u\left(t_{s}\right), v\left(t_{s}\right)$ and $u^{*}\left(t_{s}\right)$, respectively defined by

$$
\begin{gathered}
U_{h}^{s+1 / 2}=F_{1, h}\left(U_{h}^{s}\right), \quad U_{h}^{s}=c_{h}^{s}, \\
F_{3, h}^{*}\left(V_{h}^{s+1}\right)=F_{2, h}\left(V_{h}^{s}\right)+F_{3, h}\left(V_{h}^{s}\right), \quad V_{h}^{s}=U_{h}^{s+1 / 2}, \\
U_{h}^{s+1 *}=F_{1, h}\left(U_{h}^{s+1 / 2 *}\right), \quad U_{h}^{s+1 / 2 *}=V_{h}^{s+1},
\end{gathered}
$$

where $U_{h}^{s+1 *}$ represents a numerical approximation of $c_{I I}\left(t_{s+1}\right)$ and consequently of $c\left(t_{s+1}\right)$. In this algorithm $F_{1, h}$ is a linear operator resulting from the discretizations of the convection equation $(7), F_{2, h}$ represents a discretizaton of $F_{2}, F_{3, h}$ is associated with the discretization of $F_{3}$ and $F_{3, h}^{*}$ represents the discretization of the time derivative. These operators take account of the boundary conditions prescribed for (7)-(9).

\subsection{Stability}

We establish in what follows the stability of the methods described by algorithm (17)-(19). Let $c_{h}^{s+1}$ and $\tilde{c}_{h}^{s+1}$ be two solutions computed using this splitting algorithm. The difference $c_{h}^{s+1}-\tilde{c}_{h}^{s+1}$ satisfies the following equation:

$$
c_{h}^{s+1}-\tilde{c}_{h}^{s+1}=F_{1, h}\left(J F_{3, h}^{*}\right)^{-1}\left(F_{2, h}+J F_{3, h}\right) F_{1, h}\left(c_{h}^{s}-\tilde{c}_{h}^{s}\right)
$$

where $J F_{3, h}^{*}$ is the Jacobian matrix of $F_{3, h}^{*}$ computed in $\sigma V_{h}^{n+1}+(1-\sigma) \tilde{V}_{h}^{n+1}$ for some $\sigma \in[0,1]$. The Jacobian matrix $J F_{3, h}$ is defined analogously. The following proposition can be easily established:

Proposition 3 If exists $S_{C} \in(0,1)$ such that

$$
\left\|F_{1, h}\left(J F_{3, h}^{*}\right)^{-1}\left(F_{2, h}+J F_{3, h}\right) F_{1, h}\right\| \leq S_{C}
$$

then the splitting method (17)-(19) is stable.

Let us suppose now that in each step of (17)-(19) we introduce a perturbation $\epsilon_{i}$, that is

$$
\begin{gathered}
\tilde{U}_{h}^{s+1 / 2}=F_{1, h}\left(\tilde{U}_{h}^{s}\right)+\epsilon_{1} \\
F_{3, h}^{*}=F_{2, h}\left(\tilde{V}_{h}^{s}\right)+F_{3, h}\left(\tilde{V}_{h}^{s}\right)+\epsilon_{2}, \quad \tilde{V}_{h}^{s}=\tilde{U}_{h}^{s+1 / 2},
\end{gathered}
$$




$$
\tilde{U}_{h}^{s+1 *}=F_{1, h}\left(\tilde{U}_{h}^{s+1 / 2 *}\right)+\epsilon_{3}, \quad \tilde{U}_{h}^{s+1 / 2 *}=\tilde{U}_{h}^{s+1},
$$

Then we can study how these perturbations propagate from time level $t_{s}$ to time level $t_{s+1}$. Considering that

$$
c_{h}^{s+1}-\tilde{c}_{h}^{s+1}=F_{1, h}\left(J F_{3, h}^{*}\right)^{-1}\left(\left(F_{2, h}+J F_{3, h}\right)\left(F_{1, h}\left(c_{h}^{s}-\tilde{c}_{h}^{s}\right)+\epsilon_{1}\right)+\epsilon_{2}\right)+\epsilon_{3}
$$

we obtain the internal stability factor

$$
\rho_{s}=F_{1, h}\left(J F_{3, h}^{*}\right)^{-1}\left(\left(F_{2, h}+J F_{3, h}\right) \epsilon_{1}+\epsilon_{2}\right)+\epsilon_{3}
$$

which satisfies

$$
\left\|\rho_{s}\right\| \leq\left\|F_{1, h}\left(J F_{3, h}^{*}\right)^{-1}\left(F_{2, h}+J F_{3, h}\right)\right\|\left\|\epsilon_{1}\right\|+\left\|F_{1, h}\left(J F_{3, h}^{*}\right)^{-1}\right\|\left\|\epsilon_{2}\right\|+\left\|\epsilon_{3}\right\| .
$$

Attending to the last inequality we have the following proposition.

Proposition 4 If exists $I_{C} \in(0,1]$ such that

$$
\begin{gathered}
\left\|F_{1, h}\left(J F_{3, h}^{*}\right)^{-1}\left(F_{2, h}+J F_{3, h}\right)\right\| \leq I_{C} \\
\left\|F_{1, h}\left(J F_{3, h}^{*}\right)^{-1}\right\| \leq I_{C}
\end{gathered}
$$

then the splitting method is internally stable.

\subsection{Discretization errors}

We recall that $c\left(t_{s}\right)$ represents the solution of the convection-diffusionreaction equation at $t=t_{s}, c_{h}^{s}$ a numerical approximation computed using splitting method (17)-(19) and $c_{I I}\left(t_{s}\right)$ the solution computed using the functional splitting (7)-(9). We have

$$
\left\|c\left(t_{s}\right)-c_{h}^{s}\right\|_{\infty} \leq\left\|c\left(t_{s}\right)-c_{I I}\left(t_{s}\right)\right\|_{\infty}+\left\|c_{I I}\left(t_{s}\right)-c_{h}^{s}\right\|_{\infty}
$$

The first norm in the right hand side has been estimated in Proposition 1. We compute in what follows $\left\|c_{I I}\left(t_{s}\right)-c_{h}^{s}\right\|_{\infty}$. Let $T_{i, h}^{s+1}, i=1,2,3$ be the truncation errors at $t=t_{s+1}$ associated with the discretizations (17), (18) and (19) respectively. By $e_{i, h}^{s+1}, i=1,2,3$, we denote the global errors defined by $e_{1, h}^{s+1}=u\left(t_{s+1}\right)-U_{h}^{s+1}, e_{2, h}^{s+1}=v\left(t_{s+1}\right)-V_{h}^{s+1}$ and $e_{3, h}^{s+1}=u^{*}\left(t_{s+1}\right)-U_{h}^{s+1 *}$. These errors satisfy (22), (23) and (24) with $\epsilon_{i}=T_{i, h}^{s}$. Then

$$
\begin{aligned}
c_{I I}\left(t_{s+1}\right) & -c_{h}^{s+1}=F_{1, h}\left(J F_{3, h}^{*}\right)^{-1}\left(F_{2, h}+J F_{3, h}\right) F_{1, h}\left(c_{h}^{s}-\tilde{c}_{h}^{s}\right) \\
& +F_{1, h}\left(J F_{3, h}^{*}\right)^{-1}\left(\left(F_{2, h}+J F_{3, h}\right) T_{1, h}^{s}+T_{2, h}^{s}\right)+T_{3, h}^{s} .
\end{aligned}
$$


Therefore, we obtain for the truncation error of the splitting method, $T_{h}^{s}$, the following equation

$$
T_{h}^{s}=F_{1, h}\left(J F_{3, h}^{*}\right)^{-1}\left(\left(F_{2, h}+J F_{3, h}\right) T_{1, h}^{s}+T_{2, h}^{s}\right)+T_{3, h}^{s} .
$$

From the previous results and stability Propositions 3 and 4 its easy to establish the next convergence estimate for the discretization error.

Proposition 5 If (21), (25) and (26) are satisfied then

$$
\left\|c_{I I}\left(t_{s}+1\right)-c_{h}^{s+1}\right\|_{\infty} \leq \frac{1-S_{C}^{s+1}}{1-S_{C}}\left(I_{C}+1\right) \max _{i=1,2,3} \max _{\ell=1, \ldots, s+1}\left\|T_{i, h}^{\ell}\right\|_{\infty} .
$$

\subsection{Special families of methods}

Let us consider equation (1) with $z \in[0,1]$,

$$
F_{1}(c)=-\alpha \frac{\partial c}{\partial z}, F_{2}(c)=\beta \frac{\partial^{2} c}{\partial z^{2}}
$$

We discretize (7)-(9) using backward and second order centered differences respectively for first and second order space derivatives. The time derivative is discretized using the $\theta$-method which is defined by

$$
y^{s+1 / 2}=y^{s}+\frac{\Delta t}{2}\left((1-\theta) G\left(y^{s+1 / 2}\right)+\theta G\left(y^{s}\right)\right),
$$

when $y^{\prime}=G(y)$ is to be solved.

In this case we have,

$$
\left[F_{1, h}\left(U_{h}^{s}\right)\right]_{1}=\frac{1-a \hat{\theta}}{1+a}\left(U_{h}^{s}\right)_{1}
$$

and, for $i=2, \ldots, N$,

$$
\left[F_{1, h}\left(U_{h}^{s}\right)\right]_{i}=\frac{1+\hat{\theta}}{1+a} \sum_{j=1}^{i-1}\left(\frac{a}{1+a}\right)^{i-j}\left(U_{h}^{s}\right)_{j}+\frac{1-a \hat{\theta}}{1+a}\left(U_{h}^{s}\right)_{i}
$$

with $a=\alpha \frac{\Delta t}{2 h}(1-\theta), \hat{\theta}=\frac{\theta}{1-\theta}$.

For $F_{2, h}$ we have

$$
\left[F_{2, h}\left(V_{h}^{s}\right)\right]_{1}=\left(1-2 \beta \frac{\Delta t}{h^{2}}\right)\left(V_{h}^{s}\right)_{1}+\beta \frac{\Delta t}{h^{2}}\left(V_{h}^{s}\right)_{2}
$$


for $i=2, \ldots, N-1$,

$$
\left[F_{2, h}\left(V_{h}^{s}\right)\right]_{i}=\beta \frac{\Delta t}{h^{2}}\left(V_{h}^{s}\right)_{i-1}+\left(1-2 \beta \frac{\Delta t}{h^{2}}\right)\left(V_{h}^{s}\right)_{i}+\beta \frac{\Delta t}{h^{2}}\left(V_{h}^{s}\right)_{i+1}
$$

and

$$
\left[F_{2, h}\left(V_{h}^{s}\right)\right]_{N}=\beta \frac{\Delta t}{h^{2}}\left(V_{h}^{s}\right)_{N-1}+\left(1-2 \beta \frac{\Delta t}{h^{2}}\right)\left(V_{h}^{s}\right)_{N} .
$$

Finally, $F_{3, h}$ and $F_{3, h}^{*}$ are defined, $i=1, \ldots, N$, by

$$
\left[F_{3, h}\left(V_{h}^{s}\right)\right]_{i}=\Delta t \sigma f\left(\left(V_{h}^{s}\right)_{i}\right),\left[F_{3, h}^{*}\left(V_{h}^{s+1}\right)\right]_{i}=(1-\Delta t(1-\sigma)) f\left(\left(V_{h}^{s+1}\right)_{i}\right),
$$

for $\sigma \in[0,1]$.

Let us denote by Method $(\theta, \sigma)$ the splitting method just described.

It is a simple but tedious task to establish the expressions of $I_{C}$ and $S_{C}$ for Method $(\theta, \sigma)$. Let $\Delta t_{0}$ be an upper bound for the time step size. Assuming that $\left|F_{3}^{\prime}\right| \leq q$ we have:

1. For $\theta=0$ then, for $\gamma \in(0,1)$ and for $\Delta t$ small enough, we have

$$
\begin{aligned}
S_{C} & :=\gamma^{2} \frac{1+\Delta t_{0} \sigma q}{1-(1-\sigma) \Delta t_{0} q} \\
I_{S} & :=\gamma \frac{1+\Delta t_{0} \sigma q}{1-(1-\sigma) \Delta t_{0} q}
\end{aligned}
$$

2. For $\theta \in(0,1)$, there exists $K(\theta)<1$ such that, for $\Delta t$ small enough,

$$
\begin{aligned}
S_{C} & :=K(\theta)^{2} \frac{1+\Delta t_{0} \sigma q}{1-(1-\sigma) \Delta t_{0} q}, \\
I_{S} & :=K(\theta) \frac{1+\Delta t_{0} \sigma q}{1-(1-\sigma) \Delta t_{0} q}
\end{aligned}
$$

3. If $\theta=1$ then $I_{C}$ and $S_{C}$ are greater or equal to one.

Attending to the values of $S_{C}$ and $I_{C}$ we conclude that Method $(\mathbf{0}, \mathbf{1})$ and Method $(\mathbf{0}, \mathbf{0})$ are stable and internally stable. Nevertheless the stability constant of Method $(\mathbf{0}, \mathbf{1})$ is bounded by $\gamma^{2}\left(1+\Delta t_{0} q\right)$ while $\operatorname{Method}(\mathbf{0}, \mathbf{0})$ has a stability constant bounded by $\frac{\gamma}{1-\Delta t_{0} q}$.

As far as the spatial truncation error is concerned we have, for $j=1,3$, $w=u, u^{*}$,

$$
T_{j, h}^{s+1}=\frac{\partial^{2} w}{\partial t^{2}} \frac{\Delta t}{2}\left(\frac{1}{2}-(1-\theta)\right)+\alpha \frac{h}{2} \frac{\partial^{2} w}{\partial x^{2}}+O\left(h^{2}, \Delta t^{2}\right),
$$


and

$$
T_{2, h}^{s+1}=\frac{\Delta t}{2}\left(\frac{\partial^{2} w}{\partial t^{2}}-(1-\sigma) f^{\prime}\left(v\left(x_{i}, t_{s+1}\right)\right) \frac{\partial v}{\partial t}\right)+O\left(h^{2}, \Delta t^{2}\right),
$$

where the partial derivatives are evaluated at some points in $\left(x_{i-1}, x_{i}\right) \times$ $\left(t_{s}, t_{s+1}\right)$.

From Proposition 5, if Method $(\theta, \sigma)$ is stable and internally stable with stability constants $S_{C}, I_{S}$ less than one - then the discretization error $c_{I I}\left(t_{s}\right)-c_{h}^{s}$ satisfies

$$
\left\|c_{I I}\left(t_{s+1}\right)-c_{h}^{s+1}\right\|_{\infty} \leq \frac{1-S_{C}^{s+1}}{1-S_{C}}\left(I_{S}+1\right) O(h, \Delta t) .
$$

\section{Numerical examples}

\subsection{A fixed bed cathalitic reactor}

This section is concerned with the nonlinear initial boundary value problem (13)-(14) which assumes importance in chemical engineering, for instance in the modeling of fixed bed cathalitic reactors ([5]). In this model is assumed that for a given axial point, the temperature values at a characteristic position of the tube radius are representative of the whole cross section. In (13), $\gamma_{i}, i=1, \ldots, 4$, are real constants, representing the intensity of some mechanisms occurring in the system. The wall temperature $c_{w}(t)$ reflects the distributed nature of the interaction between the process and its environment and the term $r(c)$ is a nonlinear known function standing for a chemical reaction rate. In the initial-boundary condition (14), $f$ and $c_{F}$ are assumed to be known functions.

Computation have been carried with parameters:

$$
\left\{\begin{array}{l}
\gamma_{1}=-1, \gamma_{2}=10^{-3}, \gamma_{3}=-0.5, \gamma_{4}=0.5 \\
c_{F}(0)=1.1, c_{F}(t)=1, t>0 \\
r(c)=c^{2} \\
f(z)=1.11067+0.359664 z+0.325977 z^{2}, z \in(0,1) \\
c_{w}(t)=0.8, t \geq 0
\end{array}\right.
$$

In Figure 2 we plot the numerical solution computed with Method $(\mathbf{1}, \mathbf{1})$ and Method $(\mathbf{0}, \mathbf{1})$ with $h=10^{-2}$ and $\Delta t=\frac{h}{2}$

The behaviour of the numerical approximation obtained using Method $(\mathbf{0}, \mathbf{0})$ is analogous to the one presented by the numerical approximation obtained using Method (0,1). However the computational cost of Method $(\mathbf{0}, \mathbf{0})$ is greater than the computational cost of $\operatorname{Method}(\mathbf{0}, \mathbf{1})$. 

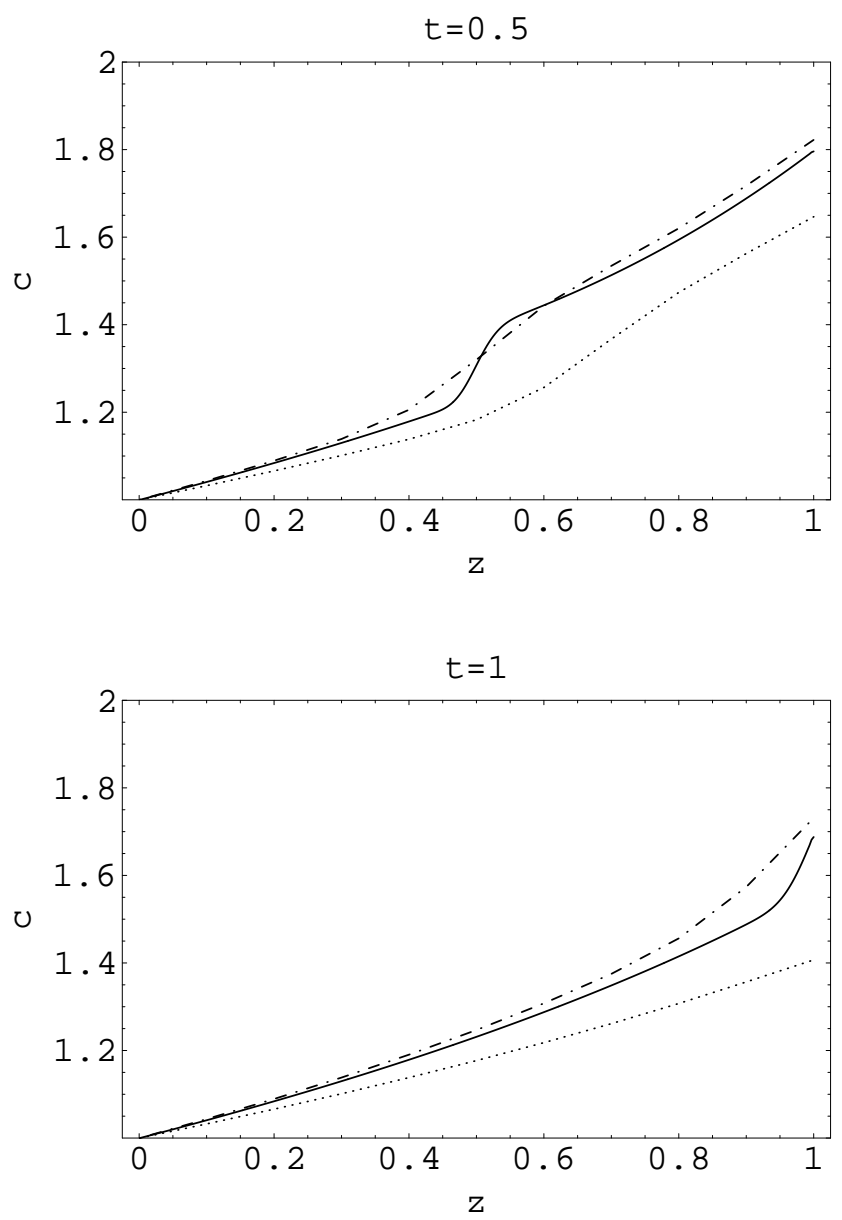

Figure 2: Numerical approximations $(\operatorname{Method}(\mathbf{1}, \mathbf{1})$; dots; Method $(\mathbf{0}, \mathbf{1})$ : dash-dots) and exact solution for (13)-(14). 


\subsection{A moving bed reactor used in the paper industry}

In this section we consider the system of hyperbolic partial differential equations that describes the behaviour of a moving bed reactor - the digester used in the paper and pulp industry $([1])$.

The digester is an heterogeneous reactor with an almost cylindrical shape, where wood chips - solid phase - react with an aqueous solution - free liquid phase - of sodium hydroxide and sodium sulfide, to remove the lignin from the cellulose fibers (Figure 3). As the wood chips are porous a third phase entrapped liquid phase - is also considered.

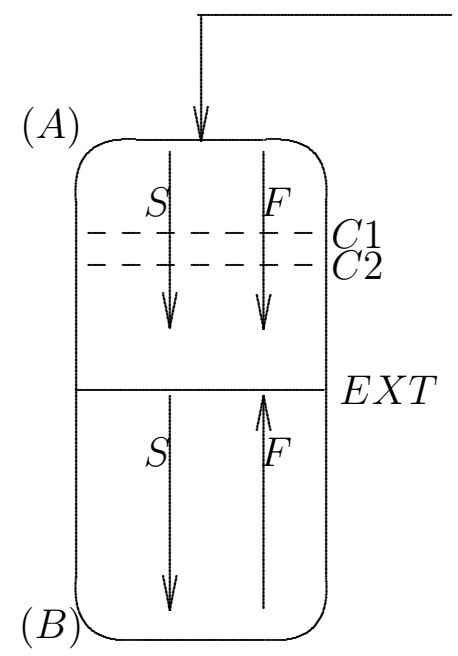

Figure 3: In the first part of the digester the phases flow concurrently while in the second part the flows are countercurrently.

From the physical point of view we can describe the behaviour of a digester as follows: (A) In its upper part the solid and the liquid phase flow downwards concurrently and at $z=E X T$ the liquid phase is completely extracted ; (B) In its lower part a free liquid flows upwards countercurrently with the solid phase ; (C) At several levels of the digester's height - the circulations $C 1, C 2$ - the free liquid is extracted, enriched and heated before being reinjected. From an industrial point of view it is important to know the temperature and the concentration of several chemical species - lignin, cellulose, hemicellulose - in the solid phase, free liquid phase and entrapped liquid phase. If $y_{i}$ denotes the concentration of some chemical product then $y_{i}^{F}, y_{i}^{E}$ and $y_{i}^{S}$ denote its concentration in the liquid, entrapped and solid 
phases respectively. For $\ell=F, E, S, y_{i}^{\ell}, i=1,2,3$, represents the concentration of respectively cellulose, hemicellulose and lignin. For $\ell=F, E, i=4,5$, $y_{i}^{\ell}$ represents the concentration respectively of solid hydroxide and solid sulfide. Finally $y_{6}^{F}, y_{6}^{E}$ stand for the temperature of the free and the entrapped liquid. The behaviour of the digester is described in a simplified way by the system of PDE's

$$
\begin{cases}\xi_{1} \frac{\partial y_{i}^{F}}{\partial t^{F}}=-u_{F} \frac{\partial y_{i}^{F}}{\partial z}+\rho_{1}\left(y_{i}^{E}-y_{i}^{F}\right), & i=1, \ldots, 5 \\ \xi_{1} \frac{\partial y_{6}^{F}}{\partial t}=-u_{F} \frac{\partial y_{6}^{F}}{\partial z}+\rho_{2}\left(y_{6}^{E}-y_{6}^{F}\right), & \\ \xi_{2} \frac{\partial y_{i}^{E}}{\partial t}=-u_{S} \frac{\partial y_{i}^{E}}{\partial z}-R_{i}-\rho_{3}\left(y_{i}^{E}-y_{i}^{F}\right), & i=1, \ldots, 5 \\ \xi_{2} \frac{\partial y_{6}^{E}}{\partial t_{T}}=-u_{S} \frac{\partial y_{6}^{E}}{\partial z}-\rho_{4}\left(y_{6}^{E}-y_{6}^{F}\right) & i=1, \ldots, 3 \\ \xi_{3} \frac{\partial y_{i}^{S}}{\partial t}=-u_{S} \frac{\partial y_{i}^{S}}{\partial z}+R_{i}, & \end{cases}
$$

where $\xi_{i}, i=1,2,3, \rho_{i}, i=1, \ldots, 4$, represent physical constants.

In system (27), the velocity $u_{F}$ is positive for $i=1,2,3$ - the liquid phase flows downwards - and is negative for $i=4,5$, and $z \geq E X T$ - because the liquid phase flows upwards countercurrently. The reaction terms $R_{i}$ are represented by exponential functions depending on $y_{i}^{\ell}$ and are experimentally established in [1]. The boundary and initial conditions as well as the values of the parameters used in the model were obtained experimentally and are established in [1].

In Figure 4 the evolution in time of the concentration of solid cellulose, solid lignin, entrapped alkali and the temperature of the free liquid are plotted. The computations have been made using Method $(\mathbf{0}, \mathbf{1})$ with constant step-size and local refinement near the circulation points.

The stationary concentrations of cellulose, hemicellulose, lignin, alkali, hidro-sulfide and the temperature are plotted in Figure 5. The results obtained for the evolution problem present physical evidence. The stationary results agree with the numerical results in [1]. 

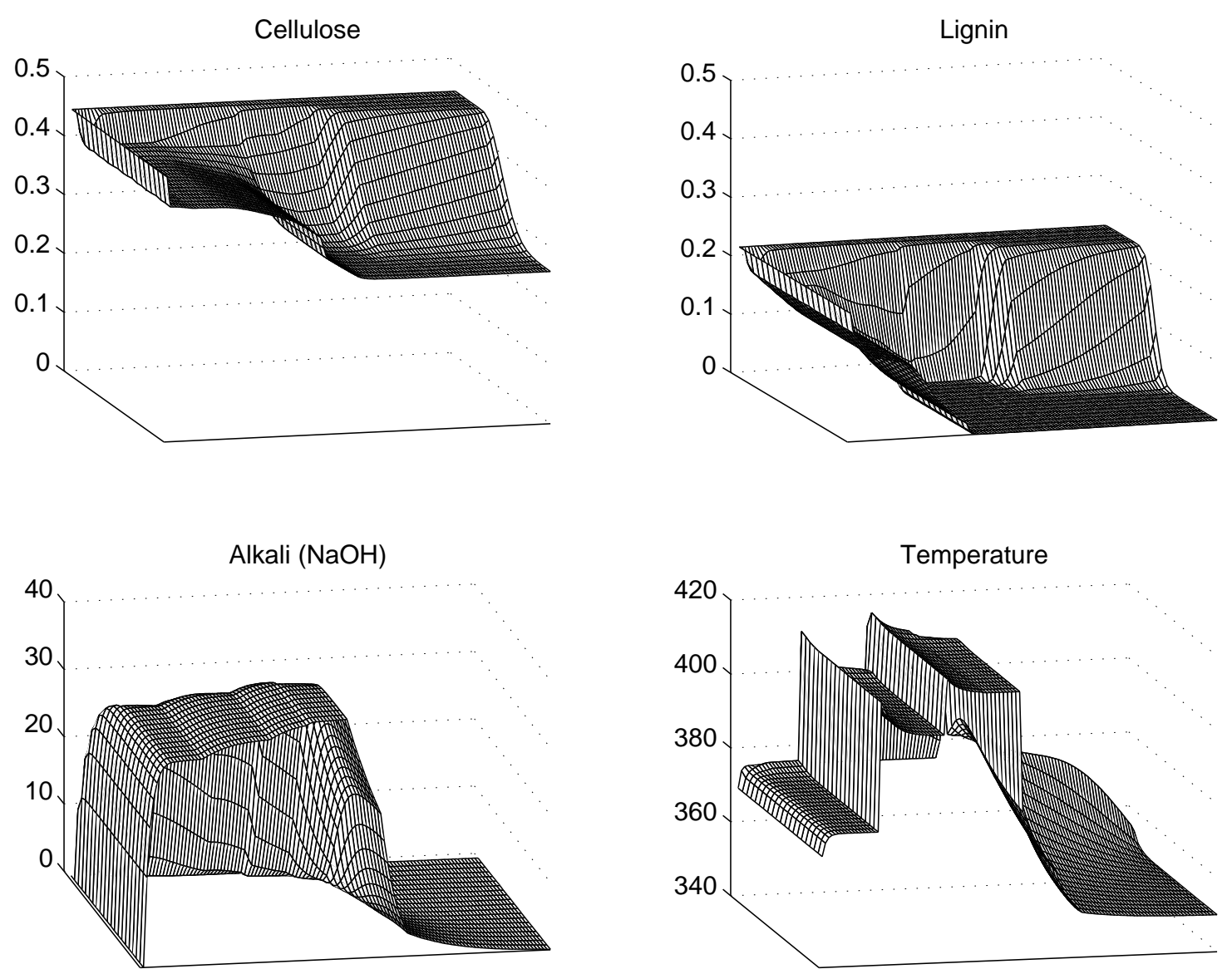

Figure 4: The evolution in time of the concentration of solid cellulose, solid lignin, entrapped alkali and the temperature of the free liquid. 

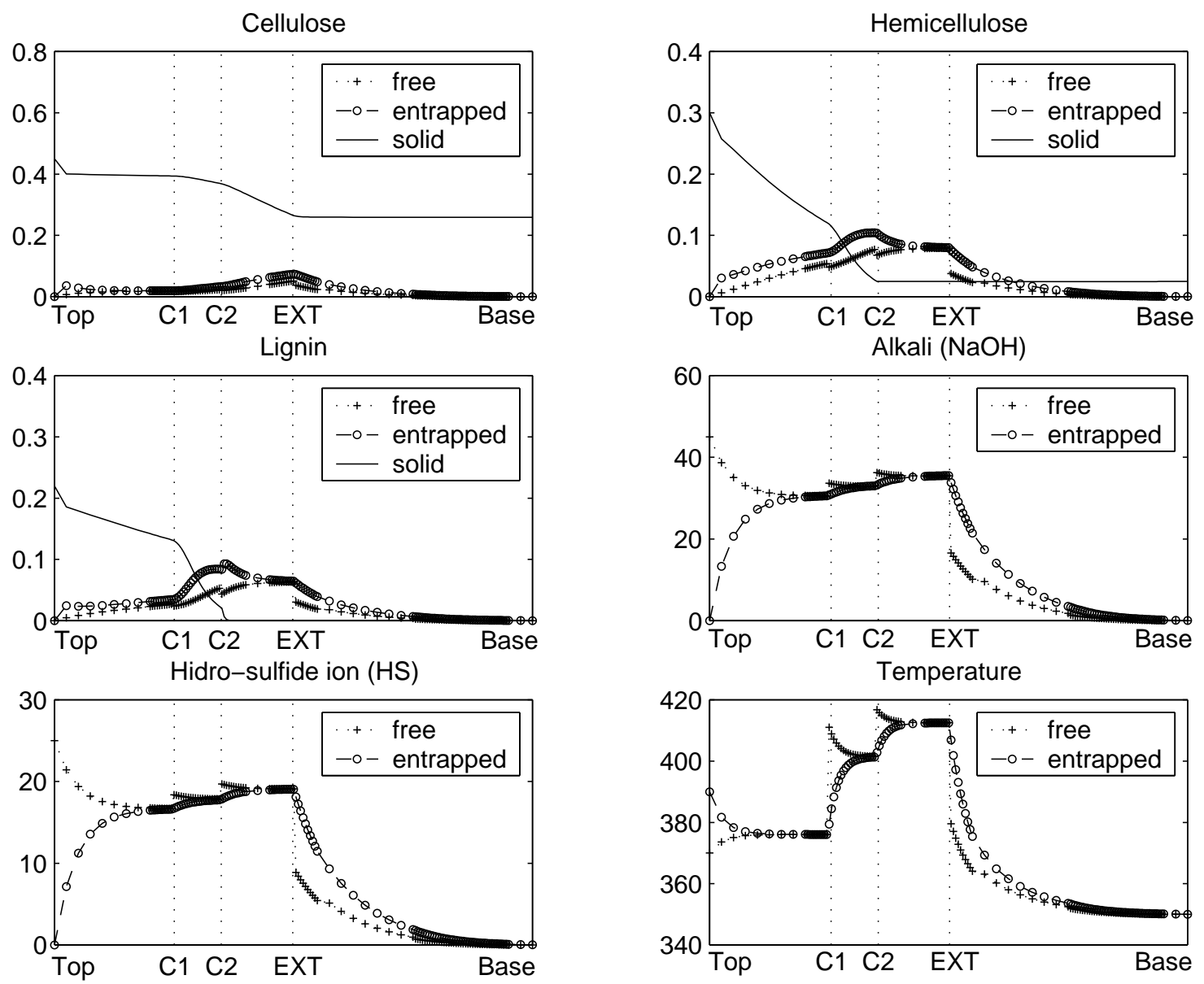

Figure 5: Numerical stationary solution for (27). 


\section{References}

[1] N.C. Fernandes, J.A.A.M. Castro, Steady-state dimulation of a continuous moving bed reactor in pulp and paper industry, Chem.Engng. Sci. $55,3729-3738,2000$

[2] W. H. Hundsdorfer, Trapezoidal and midpoint splitting for initialboundary value problems,Math. Comp. 67, 1047-1062, 1998.

[3] R.J. Leveque, Numerical Methods for Conservation Laws, BirkhauserVerlag, Basel, 1990.

[4] L.A. Monthé, A study of splitting scheme for hyperbolic conservation laws with source terms, , J. Comp. Appl. Math. 137, 1-12, 2001

[5] F. Oliveira, P. De Oliveira, M. Céu Lopes, J.A.A.M. Castro, Two adptive grid methods for fixed bed systems simulation, Computers Chem. Engng., 18, 227-243, 1994.

[6] E.J. Spee, J.G. Verwer, P.M. de Zeuw, J.G. Blom, W.H. Hundsdorfer, A numerical study for global atmospheric transport-chemistry problems, MAS- R9702, 1997, CWI, Amsterdam. 